\title{
Les villes-usines face à la problématique environnementale : réflexions à partir de l'exemple de l'agglomération dunkerquoise
}

Environmental Challenges in company towns: learning and perspective view from city of Dunkerque (France)

Die Fabriken angesichts von Umweltproblemen: Reflexionen am Beispiel der Agglomeration Dünkirchen.

\section{Christophe Beaurain et Chedrak De Rocher Chembessi}

\section{OpenEdition}

\section{Journals}

Édition électronique

URL : http://journals.openedition.org/rge/8512

DOI : $10.4000 /$ rge.8512

ISSN : 2108-6478

Éditeur

Association des géographes de l'Est

Référence électronique

Christophe Beaurain et Chedrak De Rocher Chembessi, « Les villes-usines face à la problématique environnementale : réflexions à partir de l'exemple de l'agglomération dunkerquoise », Revue Géographique de l'Est [En ligne], vol. 58/3-4 | 2018, mis en ligne le 09 mars 2020, consulté le 08 septembre 2020. URL : http://journals.openedition.org/rge/8512 ; DOI : https://doi.org/10.4000/rge. 8512

Ce document a été généré automatiquement le 8 septembre 2020

Tous droits réservés 


\section{Les villes-usines face à la} problématique environnementale : réflexions à partir de l'exemple de l'agglomération dunkerquoise

Environmental Challenges in company towns: learning and perspective view from city of Dunkerque (France)

Die Fabriken angesichts von Umweltproblemen: Reflexionen am Beispiel der Agglomeration Dünkirchen.

\section{Christophe Beaurain et Chedrak De Rocher Chembessi}

Nous remercions les deux rapporteurs pour leurs remarques qui ont contribué à une réelle amélioration du texte initialement soumis. Nous restons évidemment seuls responsables des arguments présentés dans ce texte.

\section{Introduction}

1 Apparues au cours du XIX siècle, les villes-usines se sont, au fil de la croissance industrielle, répandues un peu partout dans le monde occidental. Résultant de l'implantation d'une usine dans un espace géographique non urbanisé, elles s'accompagnent de profondes mutations dans les politiques de logement, de l'habitat et d'accessibilité aux services de base (Edelblutte, 2010a), et dans les relations entre l'ouvrier et l'usine (Picon, 2014). De Thaon-Les-Vosges (Edelblutte, 2014) aux villesusines de la moyenne Moselle (Doyen, 1983) et de la Lorraine (Picon, 2014), en passant par Le Creusot ou encore Turin (Jalabert \& Grégoris, 1987), etc., l'imbrication très forte de la ville et de l'usine est devenue une caractéristique centrale de ces territoires industriels spécifiques. Au-delà de ces configurations particulières caractéristiques de la ville-usine, ce sont plus largement l'ensemble des territoires industrialo-urbains façonnés par l'industrie qui se sont profondément transformés, dans le cadre 
notamment du capitalisme monopoliste d'État (Del Biondo, 2014). Très dépendantes de l'industrie, les villes-usines ont été confrontées à partir des années 1980 à des difficultés économiques et sociales majeures, auxquelles s'est ajoutée parfois une défaillance de planification et/ou de prospective autour de leur aménagement (Del Biondo \& Edelblutte, 2016).

2 Ces enjeux socio-économiques supposent aujourd'hui la mise en place et/ou le renforcement des politiques de reconversion urbaine et de valorisation de ces territoires autrefois attractifs. L'une des dimensions clés de la création de cette nouvelle attractivité territoriale est incontestablement la restauration de la qualité environnementale et l'insertion dans une démarche de transition écologique. Si l'enjeu de la patrimonialisation des anciens sites industriels au sein des villes-usines fait clairement ressortir l'importance des jeux d'acteurs et des innovations territoriales (Bergeron, Dorel-Ferré, 1996 ; Del Biondo \& Edelblutte, 2016), la prise en compte de la dimension environnementale s'y rattache également. Dans cette logique, et pour entrer en résonnance avec la problématique des villes en décroissance qui concerne beaucoup de ces villes-usines (Béal, Fol \& Rousseau, 2016), nous souhaitons dans cet article esquisser une réflexion sur les contours d'une dynamique collective requise pour engager les territoires urbano-industriels vers la qualité environnementale.

Pour cela, dans une première partie, cet article rappelle quelques éléments essentiels de l'évolution socio-économique et environnementale de la ville-usine. Puis de manière empirique, il s'intéresse au cas du territoire industrialo-urbain de l'agglomération de Dunkerque que nous situons précisément par rapport aux configurations des villesusines. De plus, nous relevons les interactions sociales et humaines autour de quelques démarches portées par les acteurs territoriaux de cette agglomération. Il s'agit pour l'essentiel de démarches tournées vers l'amélioration de la qualité de vie et le renforcement de l'attractivité urbaine. Enfin, dans une troisième partie conclusive, l'article tire les enseignements de l'expérience dunkerquoise. Cette dernière partie pose les bases d'une réflexion sur les innovations territoriales requises pour engager les villes-usines sur le chemin de la qualité environnementale et de la transition écologique.

\section{Villes-usines européennes : entre succès et crises}

Depuis le XIX ${ }^{e}$ siècle, des configurations spécifiques de rapports entre villes et usines ont émergé, aboutissant à des villes-usines nées de l'installation et de l'activité de grandes manufactures employant de milliers d'ouvriers (Edelblutte, 2014 ; Jalabert \& Gregoris, 1987). Ces villes-usines sont depuis quelques années marquées par un marasme social et économique, couplé à d'importantes problématiques environnementales (Luxembourg, 2008).

\section{Quand l'usine crée la ville ou l'émergence de la ville-usine}

5 Depuis le XIX ${ }^{e}$ siècle, les différentes mutations dans les modes de consommation et les organisations productives et techniques ont été à l'origine de profonds bouleversements dans les rapports entre la ville et l'industrie. Plusieurs villes ont alors accueilli des industries, en fonction des coûts comparatifs sur les matières premières, le transport, la main d'œuvre, la proximité des débouchés, etc. (Krugman, 1991, 1993). Ces 
agglomérations ouvrières, nées d'une impulsion industrielle, furent principalement celles de la mécanique en Allemagne, du textile et de la sidérurgie en Grande-Bretagne et en France (Verley, 2016). Elles associèrent l'urbanisation ou la transformation rapide de bourgades en villes à l'implantation d'une firme industrielle. En France, la commune lorraine de Thaon-les-Vosges est l'archétype d'un territoire construit par l'industrie, notamment d'un système productif industriel paternaliste. En effet, comme le souligne S. Edelblutte (2014), l'installation d'une entreprise en 1872 avec quelques centaines d'ouvriers sur une commune qui ne comptait alors que 555 habitants, a profondément bouleversé le système spatial avec la construction de cités ouvrières, et une ville progressivement tournée vers l'activité industrielle. Cette urbanisation est à mettre en perspective avec le développement des services de base dans les villes-usines, tels les hôpitaux, les écoles, les centres de loisirs, des jardins ouvriers, etc., porté par les firmes dominantes (Edelblutte, 2010a ; Doyen, 1983). Et contrairement à la ville industrielle, qui renvoie à une ville préexistante ayant accueilli ensuite des industries, la ville-usine apparaît comme un système spatial entièrement ou presque né d'une ou de plusieurs usines, principalement du secteur minier. C'est le cas des villes préindustrielles du nord de la France dont l'organisation urbaine a été profondément transformée par l'implantation d'une ou de quelques firmes d'un secteur de l'industrie (Edelblutte, 2010b). Dans l'organisation des rapports entre ville et industrie qui s'est imposée, la ville a été véritablement restructurée par l'industrie dans toutes ses dimensions socioéconomiques essentielles. Ce qui autorise à parler en termes de «territoires industrialo-urbains " (Del Biondo, 2014). Dans ces territoires, l'usine a "modelé, et fabriqué des citoyens en même temps que s'élevaient les hauts-fourneaux " (Aubert, 2001), mais aussi le paysage d'un espace géographique (Del Biondo \& Edelblutte, 2014; Fortin \& Gagnon, 2006; Ryan \& Campo, 2013)

6 Cependant, depuis la fin du $\mathrm{XX}^{\mathrm{e}}$ siècle, frappées comme l'ensemble des villes industrielles par la crise des secteurs traditionnels de l'industrie, les villes-usines sont devenues le théâtre de tensions sociales souvent accompagnées d'une dégradation de la qualité environnementale et d'une multiplication des friches industrielles (Raggi, 2019 ; Bacqué 2018). Pour les plus critiques, ces tensions et dégradations seraient la conséquence d'une urbanisation sauvage avec des agglomérations sans aucun plan d'ensemble, des logements contigus presque indécents et malsains, avec des travaux de voirie insuffisants, une forte pollution de l'air, une forte pression de la demande de logement par rapport à l'offre, etc. (Edelblutte, 2009). Dans ces conditions, la question environnementale apparaît souvent comme un enjeu emblématique, tant par l'ampleur de la dégradation de l'environnement que par l'importance prise par la construction d'un nouveau rapport à la nature ou à l'environnement dans les perspectives de sortie de crise.

\section{Les problématiques environnementales dans les villes-usines en crise}

$7 \mathrm{Au}$ cœur des premières vagues d'industrialisation, les villes-usines constituent un système industriel reposant sur les mines, la métallurgie, l'électricité, la chimie de synthèse, exploitant abondamment des ressources naturelles (Verley, 2016). Ces secteurs d'activités sont symptomatiques de l'échec sur bien des plans, notamment sociologique (crise des rapports sociaux), urbanistique (manière de penser la ville) et environnemental, de la dynamique industrialo-urbaine du $\mathrm{XX}^{\mathrm{e}}$ siècle. En plus de 
l'effondrement de la rentabilité, l'endettement des entreprises, une faible croissance de la production industrielle, des suppressions massives d'emplois (Teulon, 2010), de nombreux défis sociaux et environnementaux ont jalonné le déclin des villes-usines, vérifiant à postériori la thèse prévisionnelle de Toynbee (1884), ou plus près de nous celle de Mumford (1964), qui faisaient des métropoles industrielles et/ou des espaces usiniers des « bombes à retardement $»^{1}$.

\section{Des crises de pollutions et un rapport ville-nature détérioré.}

Parallèlement à leur intégration dans une forte dynamique économique, les villesusines ont-elles-constitué également des chantiers de destruction socioenvironnementale ? Bien que la réponse à cette question ne paraisse pas évidente de prime abord, on peut néanmoins souligner que ces villes ont été marquées par d'importants rejets de polluants sans traitement, et plus globalement par une détérioration de l'environnement naturel (Luxembourg, 2008; 2014). Rien ne semble en effet indiquer que les villes-usines aient pu échapper au phénomène, observé dans l'ensemble des territoires industrialo-urbains, de dégradation de l'environnement naturel par les procédés industriels. La ville-usine de Norilsk (Russie), fondée au début des années 1930 avec l'un des plus grands centres industriels métallurgiques dans le monde encore aujourd'hui et façonnée par l'exploitation des minerais de la Sibérie, s'est longtemps distinguée par la mauvaise qualité de l'environnement. On peut souligner une très forte concentration de cuivre et de nickel dans les sols, d'importantes émissions de gaz à effet de serre ayant généré des taux élevés de maladies respiratoires, notamment chez les enfants. En France, l'histoire de la ville du Creusot, souvent qualifiée de ville-usine ${ }^{2}$, se conjugue aussi avec la disparition progressive du paysage naturel. Comme le soulignent Lebrun et Lasseray (1936), les produits rejetés par les cheminées des usines ont généré dans cette ville une détérioration de la qualité de vie due à la pollution de l'air, de l'eau, ainsi que d'importantes modifications paysagères. En effet, les villes-usines se sont développées bien avant le renforcement de la législation environnementale mise en place à partir des années 1970 pour limiter les impacts de l'industrie sur l'environnement naturel. En analysant le rapport ville-nature dans les processus d'industrialisation, l'aménagement des espaces industriels a contribué à réduire les aménités environnementales (Beaurain, 2014). Et cette rupture entre la ville et la nature a, d'une certaine manière, participé au déclin de l'attractivité des territoires industrialo-urbains. Ainsi, bien que les usines aient généré l'émergence et la croissance urbaine, leur évolution depuis le $\mathrm{XIX}^{\mathrm{e}}$ siècle s'est faite sans prise en considération du rôle de la nature dans la construction du rapport entre l'Homme et son milieu de vie. Par ailleurs, ces espaces urbains spécifiques furent dénoncés comme le lieu d'expression de fortes disparités sociales, notamment dans l'habitat ouvrier.

\section{Des limites sociales de la ville-usine}

9 Dans les villes-usines, l'essentiel de la politique sociale des industriels a consisté à construire des cités ouvrières, sur le modèle d'une reproduction standardisée d'habitats de taille réduite et pavillonnaires. On peut ici souligner le déplacement de l'agglomération urbaine du Creusot vers le sud, au fur et à mesure que s'élevaient les fourneaux industriels. Cela a eu pour conséquence «des maisons serrées et noircies dans le centre-ville ", et des "cités industrielles avec leurs îlots réguliers de maisons 
entourées de jardins" (Lebrun, Lasseray, 1936, p.185). L'âge d'or de l'histoire industrielle de Joeuf, ville-usine dont le développement est fortement marqué par les mines de fer et l'expansion sidérurgique, est aussi celui d'un bouleversement du paysage urbain. En effet, après l'usine en 1880, des cités ouvrières, des commerces et de nouvelles routes ont profondément transformé le village d'origine en une véritable cité industrielle cosmopolite vers les années $1914^{3}$.

Et depuis quelques années, frappés par la crise, ces territoires sont confrontés à la problématique des "villes rétrécissantes » ou « en décroissance » (Fol \& CunninghamSabot, 2010). Celle-ci est symbolisée à la fois par la multiplication des terrains vacants (friches industrielles, destruction des anciens logements ouvriers...), le vieillissement de la population, la stagnation voire le recul démographique, l'appauvrissement, et la difficulté à créer une nouvelle dynamique économique autour d'activités et de secteurs connectés aux réseaux mondiaux créateurs d'emplois, soulignant de fait la fragilité de ces espaces urbains (Roth, 2018). Ce constat laisse à penser que les villes-usines pourraient être confrontées, dans leurs politiques de renouveau urbain et économique, au passage d'une approche purement quantitative (croissance de la population et de l'emploi) à une politique plus qualitative, centrée sur l'amélioration de la qualité de vie des habitants, éventuellement axée vers une acceptation de la gestion de la décroissance (Béal, Fol, Rousseau, 2016). Dans cette logique, la question des stratégies foncières concernant les terrains vacants ou la ré-affection du bâti industriel existant à d'autres fins qu'industrielles paraît alors centrale ${ }^{4}$. Ces stratégies se conjuguent avec des politiques locales de restauration de la qualité environnementale et d'engagement dans une démarche de transition écologique. C'est précisément pour ouvrir une réflexion sur ce point que nous mobilisons maintenant l'exemple de l'agglomération dunkerquoise, en considérant ici l'importance de la problématique environnementale pour les villes-usines dans leurs efforts pour surmonter les effets de la crise.

\section{L'agglomération dunkerquoise : De la ville façonnée par l'industrie au renouveau urbain.}

\section{Croissance urbaine et « sidérurgie sur l'eau »}

L'agglomération dunkerquoise ${ }^{5}$, à l'extrême nord du littoral de la Côte d'Opale, présente la particularité de cumuler la présence d'un site industriel encore fortement prégnant ${ }^{6}$ en dépit des crises économiques successives qu'il connaît depuis les années 1980, l'existence d'un site balnéaire (Malo-les-Bains, Bray-Dunes prolongé par le site belge de De Panne) et un littoral aux attraits environnementaux réels, notamment pour un tourisme de proximité ${ }^{7}$. Le tissu économique local regroupe plusieurs établissements industriels dont la plupart sont de gros émetteurs de polluants atmosphériques $^{8}$ et génèrent d'importants impacts environnementaux. À cet effet, l'agglomération a intégré depuis plusieurs années la problématique du développement durable dans sa politique territoriale, avec l'objectif affiché de concilier industrie et environnement naturel. 
Figure 1 : L'industrie dans l'agglomération de Dunkerque

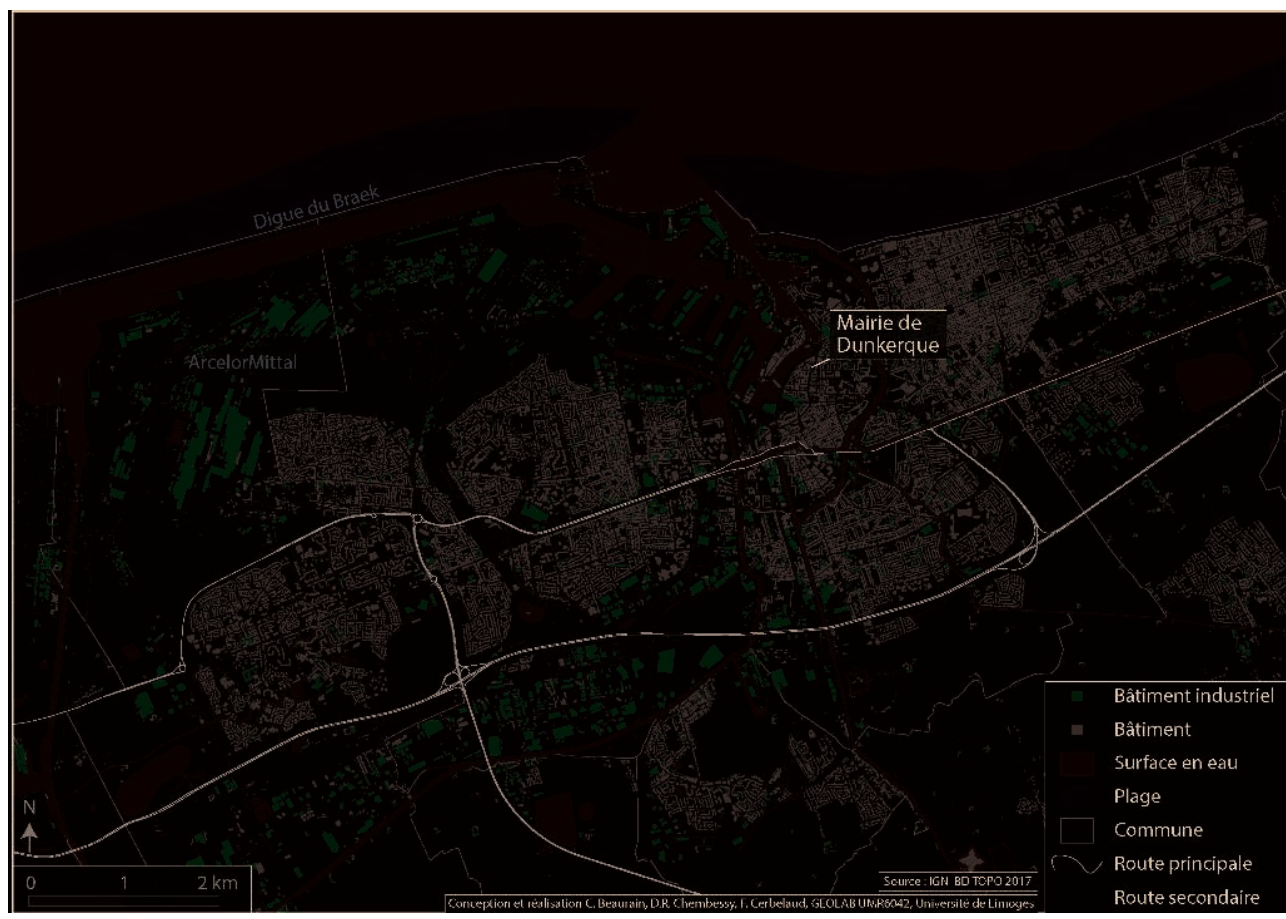

Même si l'on ne peut parler stricto sensu de ville-usine à propos de l'agglomération dunkerquoise, la comparaison avec ce type d'espace urbain paraît à bien des égards justifiée. La carte 1 illustre la forte présence des unités industrielles sur le territoire dunkerquois, l'empiètement sur le littoral qu'une partie d'entre elles a réalisé, et l'encerclement de l'agglomération par les bâtiments industriels. Cependant, l'agglomération de Dunkerque relève davantage de l'historique des villes industrielles classiques que de celle des villes-usines. En effet, l'industrie s'y est implantée et développée dans la seconde moitié du XX $\mathrm{X}^{\mathrm{e}}$ siècle plutôt en dehors des limites de l'espace urbain. Par ailleurs, celle-ci a bénéficié d'un soutien politique pour une bonne partie des fonctions socio-urbaines liées à la croissance de la main-d'œuvre. Néanmoins, plusieurs autres éléments du développement de ce territoire industrialo-urbain invitent à suggérer une réelle proximité avec les villes usines. En premier lieu, l'espace urbain dunkerquois s'est tout au long de son histoire organisé autour des infrastructures portuaires ${ }^{9}$. Le port se distingue alors comme l'un des espaces centraux de la ville et l'un des moteurs du développement économique. Ensuite, l'implantation de la sidérurgie à partir de la fin des années 1950 et son développement jusqu'aux années 1970 a complètement façonné un nouveau système spatial. La structuration socio-spatiale de la ville en a été profondément transformée. On peut souligner la création d'une agglomération, intégrant les zones industrielles et l'extension de la zone portuaire au-delà des limites traditionnelles de la ville, et la création en 1968 de la première Communauté Urbaine volontaire. La grande entreprise a, avec l'aide appuyée de l'État et dans le cadre du capitalisme monopoliste d'État ${ }^{10}$, influencé très fortement les choix en matière d'organisation socio-urbaine, dans la logique des "opérations marquantes » de l'aménagement sous l'ère gaullienne (Castells \& Godard, 1974).

De la fin des années 1950 jusqu'au début des années 1970, le développement économique local repose à la fois sur la croissance de l'activité portuaire et sur la constitution d'une activité sidérurgique dominante au sein du tissu industriel local. Le 
démarrage de l'entreprise Usinor (aujourd'hui Arcelor-Mittal) en 1962 marque le début d'une spécialisation du territoire dans la métallurgie, qui perdure encore aujourd'hui. Dès les années 1960, la firme sidérurgique est rapidement devenue l'entreprise dominante sur le territoire. Elle participe à la création des emplois, et exerce une influence technique et économique sur les sous-traitants ou le secteur portuaire ${ }^{11}$. Outre le développement industriel et portuaire massif qu'elle a favorisée ${ }^{12}$, cette implantation sidérurgique a profondément bouleversé le paysage urbain et environnemental de l'agglomération, entrainant à la fois une défiguration du paysage littoral et une croissance urbaine sans précédent. La construction du complexe sidérurgique, sur une surface totale de 450 hectares (dont 85 gagnés sur la mer), premier exemple en France de sidérurgie sur l'eau, a été réalisée sur un espace composé principalement de massifs dunaires et de plages, par lesquels les habitants accédaient auparavant à la mer, et de quelques terrains cultivés ${ }^{13}$. Une digue (la « digue du Braek») de $7 \mathrm{~km}$ de long a également été érigée pour protéger l'entreprise des marées, et créer un bassin maritime facilitant l'accostage des navires minéraliers (tirant d'eau maximum de 2,5m), dans le cadre plus général de l'extension des équipements portuaires sur une vingtaine de kilomètres à l'ouest de l'agglomération.

Cette artificialisation du littoral et des terres agricoles environnantes s'est accompagnée à la fois d'une réelle dynamique économique, et d'une très forte croissance urbaine ${ }^{14}$. Une bonne partie des équipements industriels et portuaires (zones industrielles, et des infrastructures de transports à partir des années 1960 ont été construits dans le but de répondre aux besoins de l'industrie sidérurgique (Castells, Godard, op.cit.). En outre, les rapports sociaux internes à l'agglomération ont été complètement bouleversés, avec notamment l'apparition d'une bourgeoisie associée au secteur monopoliste intégré (issue notamment des corps d'ingénieurs) et de classes dirigeantes qui ont progressivement supplanté, dans les instances décisionnelles politiques et socio-économiques de l'agglomération, la petite bourgeoisie portuaire historiquement dominante. Cette nouvelle classe sociale se caractérisait par des rapports étroits avec le capital bancaire et l'État. Ce bouleversement a également été favorisé par le développement d'une population ouvrière partie prenante de ce secteur monopoliste intégré, souvent d'origine extérieure au territoire (venant notamment du sud-est du département du Nord, ou encore de Lorraine), et confrontée pendant de longues années à des problèmes de logement. Une urbanisation à marche forcée (6000 logements créés entre 1962 et 1982, par le biais notamment de la création de ZUP et ZAC) ${ }^{15}$ a ainsi été enclenchée avec l'appui de l'Etat pour faire face aux "goulots d'étranglement » dans ce domaine du logement. L'entreprise sidérurgique a fortement pesé dans les choix d'implantation des logements à proximité du site industriel. En revanche, la qualité du cadre de vie des logements proposés aux nouveaux salariés du site est restée, durant toute cette période, une question peu prise en compte dans les priorités d'alors. Castells et Godard soulignent ainsi que la grande entreprise a joué un rôle décisif dans la production et la gestion du système urbain et que le "nouveau Dunkerque [a été] produit par Usinor ", au sens où « la ville a été l'unité à travers et par laquelle la force de travail mise en valeur vit, se reproduit et agit socialement » (1974, p.174). De manière générale, c'est toute l'organisation de la planification urbaine, au travers notamment de l'influence de l'Etat et de l'entreprise sidérurgique sur les orientations privilégiées dans les documents d'urbanisme, qui a été soumise à la logique de la grande industrie. Comme en témoignent les nombreux conflits avec les 
acteurs locaux sur ce point au moment de la réalisation du Schéma directeur d'aménagement et d'urbanisme (Ibid.).

\section{Déclin industriel et revendications environnementales : l'émergence d'un projet collectif de territoire au tournant des années 90}

La réduction drastique des effectifs dans la sidérurgie à partir des années $70^{16}$ et la disparition des chantiers navals en 1987 ont contraint les acteurs locaux à se lancer dans une politique de diversification des emplois, fondée sur le renforcement de l'attractivité du territoire. Cette évolution économique s'est inscrite dans le cadre d'un projet de territoire, élaboré au début des années 1990 et mis en œuvre à travers les actions programmées dans les documents de la planification territoriale. Deux dimensions principales ressortent clairement de ce projet : la rénovation urbaine, tout d'abord, par la restauration d'une continuité entre la ville et le port et la réhabilitation des quartiers en difficulté; la conciliation du développement industriel et de l'amélioration de la qualité de vie, ensuite.

\section{La rénovation urbaine : réconcilier ville et port.}

Les friches laissées par la fermeture des chantiers navals en 1987 et la dégradation de l'habitat collectif construit en hâte dans les années 1960 ont incité les autorités politiques locales à mettre en place un véritable projet de rénovation urbaine, progressivement inséré dans une démarche plus large de développement durable urbain. Le projet urbain a notamment concerné la requalification des friches portuaires au sein de la ville de Dunkerque. La volonté affichée consiste à recréer un continuum urbanisé entre le centre-ville et le port, et plus généralement entre la ville et la mer, et à réintroduire la nature dans la ville. Le projet, baptisé « Neptune », réalisé en deux phases successives, a eu deux objectifs principaux. Durant la première phase, l'action a porté sur la rénovation des bassins du vieux port (par la création d'une zone commerciale située entre le centre-ville et le port et par l'implantation de bâtiments de l'université). Il s'agissait ici de recréer une centralité et une citadinité au sein de la ville. Dans une deuxième phase, Neptune 2 (requalification du site des anciens chantiers navals pour la construction de logements privatifs et collectifs), avait pour objectif de réaliser un écoquartier (Écoquartier du Grand Large) et d'utiliser des anciens bâtiments pour créer un musée (FRAC Grand Large et des Hauts de France). Il a ainsi permis de revaloriser les bassins, issus des anciennes zones portuaires, situés en cœur de ville. Et à partir des années 2000, une politique de constitution d'une trame verte / trame bleue est engagée à l'échelle de l'agglomération. Outre les espaces de nature " classiques ", elle intègre également des espaces classés en Natura 2000 « mixte : terre et mer » et en Natura 2000 "mer ». En dépit de l'industrialisation massive des années 60-70, la nature n'a jamais cessé d'exister. Les plages et les digues de mer (station balnéaire de Malo-les-Bains et digue du Braek), ont largement été appropriées par les habitants pour des usages récréatifs (sports de voile, sports de plage, pêche...) et la recherche d'un contact quotidien à la nature. La présence des espaces dunaires protégés contribue également à la qualité et au caractère sauvage du paysage naturel. La carte 2 (ci-dessous) témoigne de cette restauration du lien avec la nature, au cœur même de l'agglomération. Cette régénérescence de la nature en ville s'est souvent construite à des endroits stratégiques, notamment à proximité de secteur à forte 
densité de population, des plages notamment de l'extrême Est de la digue de Malo-lesBains, et à partir d'une importante mobilisation territoriale.

Figure 2 : Présence de la nature au sein d'un territoire industriel comme Dunkerque

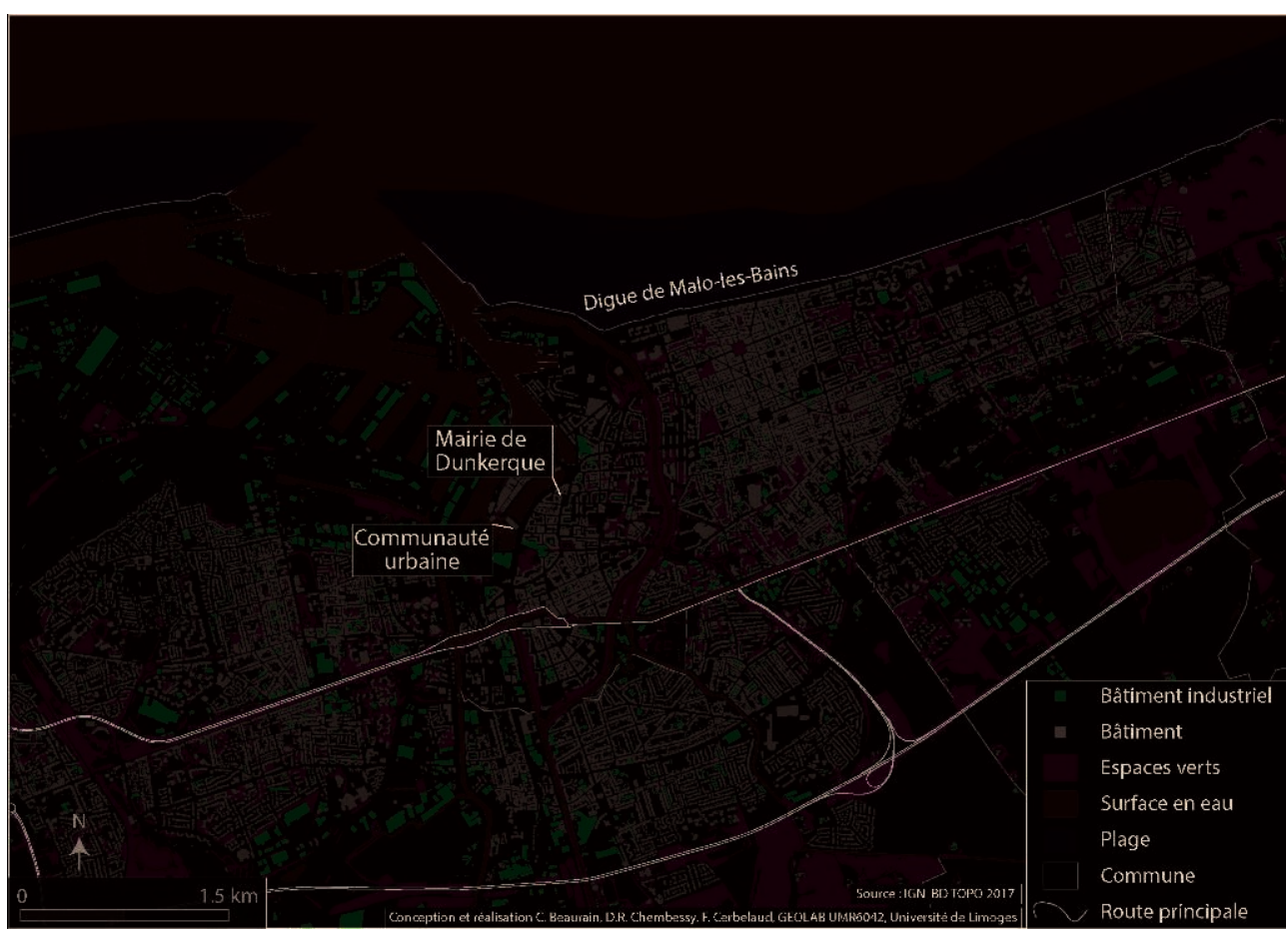

Cette présence d'espaces naturels à proximité de la ville ou en son cœur même invite à interroger le rapport des habitants à la nature, et particulièrement au littoral. De plus, elle traduit le fait que la forte prégnance de l'industrie sur le territoire n'est donc pas exclusive de l'importance de la sensibilité et des pratiques des habitants à l'égard des diverses formes de la nature en ville. ${ }^{17}$

\section{La question centrale de la qualité de l'air et du risque industriel}

La crise économique et la prise de conscience environnementale dans les années 1990 ont favorisé l'émergence d'une demande croissante de la population en faveur d'une amélioration de la qualité de vie et d'une réduction des impacts environnementaux de l'industrie. Pour répondre à cette demande, un véritable projet de territoire partagé va émerger au fil des documents de planification territoriale, contribuant à la construction d'une action collective autour de la notion d'« environnement industriel » (Beaurain, 2008). Différents documents indiquent clairement les objectifs des acteurs publics locaux dans ce domaine ${ }^{18}$. Du côté de la population, la demande sociale en faveur de l'environnement porte sur la qualité de l'air et la réduction significative des rejets industriels. Les conflits, croissants et de plus en plus durs, entre les industriels, les habitants, et les représentants des associations de défense de l'environnement ont ainsi convaincu les autorités politiques locales de la nécessité d'engager des actions dans ce domaine. Les dispositifs institutionnels mis en place pour la conciliation du développement industriel et la qualité environnementale ${ }^{19}$ témoignent de l'émergence d'une action collective territorialisée (Beaurain et allii, 2006). De plus, l'importance attachée à la perception de la pollution atmosphérique et à la gestion territoriale du 
risque industriel au sein de l'agglomération (Beaurain et allii, 2009) renvoie à une volonté manifeste de reconstituer un paysage urbain fortement affecté par les émissions atmosphériques de l'industrie.

Cette démarche territoriale permet de souligner que repositionner la question environnementale présente des difficultés pour faire émerger des représentations sociales partagées du risque industriel. Ces difficultés se traduisent notamment par une différence de compréhension des enjeux, et des valeurs de référence de chacun des acteurs (Beaurain et allii, 2009). Cependant, un objectif commun se dégage au travers des délibérations collectives régulières, souvent très conflictuelles, à propos des différentes valeurs attachées à la qualité de l'environnement. Dans le cas dunkerquois, il porte sur la limitation des impacts sanitaires, la défense de la qualité de l'environnement, une meilleure attractivité économique, une plus grande qualité de vie et, pour certaines entreprises, l'amélioration des processus de production. Toutefois, il paraît difficile de parler de "concertation », bien que beaucoup d'acteurs soulignent l'intérêt des processus d'apprentissage réciproque qui se développent au sein de ces structures. Ajoutons néanmoins que le rôle des pouvoirs publics dans ces dispositifs de restructuration par le prisme environnemental paraît mitigé. En effet, ils apparaissent à la fois comme des défenseurs du pouvoir économique des firmes, et comme des acteurs majeurs du processus d'apprentissage réciproque. De manière générale, cette question de la qualité de l'air révèle l'importance de la pérennisation d'une bonne gouvernance entre les acteurs impliqués, constamment remise en chantier.

\section{La constitution d'une symbiose industrielle au sein de l'agglomération dunkerquoise}

C'est dans le contexte d'industrialisation rapide et massive du territoire à partir des années 1960 que les premières synergies s'apparentant à des pratiques d'écologie industrielle vont se mettre en place. Et celles-ci l'ont été bien avant que l'écologie industrielle ne devienne un champ de réflexion à part entière. Sur le territoire dunkerquois, les premiers échanges de flux de matières et d'énergie entre firmes sont exclusivement liés au processus de production de la firme sidérurgique dominante (Usinor). Si ces premiers échanges ont permis la réutilisation des sous-produits d'acier, notamment « le laitier » dans la construction routière, et la valorisation des gaz issus des procédés sidérurgiques en électricité, ceux-ci ne l'ont pas été à proprement parler pour des raisons écologiques mais en vue d'une optimisation de la chaîne productive. L'intérêt écologique n'apparaît réellement qu'avec l'association des flux issus du process sidérurgique à un réseau de chauffage public.

Cette première phase, limitée à des acteurs privés, a pris un nouvel essor avec l'entrée en jeu de la Ville de Dunkerque en 1983, dans le cadre du projet de réutilisation de la chaleur fatale $e^{20}$ issue du process du sidérurgiste à destination du chauffage urbain. Le réseau ainsi créé atteindra près de $70 \mathrm{~km}$ à partir de 1985, en reliant les chaînes d'agglomération de la firme à des logements HLM, des bâtiments publics, puis plus récemment des logements privés. Cette synergie a eu des impacts significatifs sur les rejets de la firme sidérurgique dans l'atmosphère $\left(\mathrm{CO}_{2}\right.$ et poussières), tout en assurant le chauffage pour des bâtiments publics, des bureaux et des logements privés, collectifs et individuels. ${ }^{21}$ Dans les années 1990, parallèlement au contexte territorial incitant à un meilleur appariement entre le développement industriel et la qualité de l'environnement, de nouveaux projets relevant de l'écologie industrielle et impulsés 
par les grandes firmes du territoire dans le cadre de relations bilatérales se sont développés aboutissant à une symbiose industrielle (Varlet, 2012). Permettant de créer de la valeur pour les entreprises et les territoires de projet, une symbiose industrielle renvoie à un réseau de synergies et d'échanges, particulièrement de résidus de matières, d'énergies, d'eaux ou de ressources entre des acteurs industriels et/ou des collectivités dans un espace géographique donné. Ce réseau d'échange s'appuie sur des collaborations entre entreprises, et différents acteurs partenaires dont la mobilisation, les interactions, la coordination, etc. permettent de porter un projet innovant pour le territoire (Chertow, 2007).

Dans l'agglomération de Dunkerque, l'un des éléments déclencheurs de l'élargissement du réseau d'acteurs a incontestablement été la création en février 2001 d'une structure chargée de promouvoir l'écologie industrielle. En effet, la création de l'association Ecopal marque un tournant important dans l'essor de l'écologie industrielle à Dunkerque. En effet, elle illustre une volonté d'élargir une démarche reposant jusque-là sur des échanges bilatéraux et spontanés à une dimension collective et planifiée. S'appuyant sur le potentiel local, les synergies industrielles se sont principalement bâties autour de l'activité sidérurgique: laitiers sidérurgiques, valorisation des gaz, chauffage urbain et valorisation de briques réfractaires. De plus, on observe à la fois un approfondissement des engagements entamés dès les années 1960 et l'émergence de nouvelles filières de valorisation (filières agricoles pour l'épandage, filière des cimentiers, recyclage des briques réfractaires des sidérurgistes à des fins de réutilisation de la matière première ou de production d'alumine, recyclage des poussières des sidérurgistes...). En définitive, la symbiose industrielle de Dunkerque met en évidence un ensemble d'entreprises et d'acteurs territoriaux liés par des objectifs ou intérêts communs, qui mènent une action collective tout en conservant une identité et un statut qui leur sont propres (Varlet, 2012). Inscrite très tôt dans la problématique du développement durable ${ }^{22}$, l'agglomération dunkerquoise a poursuivi cet engagement avec l'adoption en 2009 d'un Plan climat territorial (2009-2014), avant même l'obligation réglementaire imposée par le Grenelle II de l'environnement en 2010. L'élaboration de ce plan a connu la mobilisation d'environ 200 acteurs locaux à travers des groupes de travail thématiques, et qui a débouché sur des chartes d'engagement déclinées par acteurs (Beaurain, Frère, Flanquart, 2017). Avec l'adoption en 2015 d'un nouveau Plan Climat Territorial, l'agglomération de Dunkerque mise sur l'implication de différents acteurs locaux autour de divers projets relevant des synergies industrielles (comme le développement du terminal méthanier par exemple), pouvant contribuer à l'attractivité du territoire. Ce détour par le territoire urbanoindustriel dunkerquois montre l'importance de reconstruire le lien entre la ville et la nature d'une part, et de concilier l'économie et l'écologie d'autre part, dans la quête du renouveau urbain des anciens territoires urbano-industriels. En guise de réflexion conclusive, nous essayons dans la section suivante de tirer les enseignements de l'exemple dunkerquois pour les villes-usines. 


\section{Quels enseignements de l'expérience dunkerquoise pour la reconversion des villes-usines face à la problématique environnementale?}

23 L'agglomération dunkerquoise ne peut à proprement parler être assimilée à une villeusine, du type des villes minières ou sidérurgiques. Néanmoins, les transformations urbaines induites dans cette ville par le développement industriel à partir des années 1960, sous l'influence du secteur intégré dans le capitalisme monopoliste d'État, renvoient incontestablement aux territoires urbano-industriels. En effet, l'agglomération s'inscrit dans cet ensemble urbain dont l'aménagement et la vie sociale ont été placés sous l'influence directe des acteurs industriels et de certains opérateurs énergétiques, comme EDF par exemple. À ce titre, le cas dunkerquois est intéressant pour la compréhension des enjeux environnementaux auxquels sont désormais confrontées les villes-usines dans leur stratégie d'attractivité. On peut plus précisément tirer deux enseignements majeurs du cas dunkerquois.

En premier lieu, la reconstruction socio-politique d'un nouveau rapport ville - nature apparaît comme une dimension essentielle de l'amélioration de la qualité de vie. Si les bouleversements de l'environnement naturel dans les années de croissance économique et de développement industriel ont été impulsés par le secteur monopoliste d'État, sa reconquête à partir des années 1980 relève principalement d'une prise en charge par la communauté locale. De ce point de vue, il faut insister sur l'importance d'une volonté, exprimée à la fois par le pouvoir politique (dans les actions d'aménagement) et par les habitants (dans leurs revendications en faveur de la limitation des pollutions), de restaurer le milieu de vie identitaire, le rapport à la mer et aux paysages qui le composent (Beaurain, 2014). Cette volonté a marqué un renversement notable des rapports entre l'industrie et la communauté locale sur la question de l'aménagement, en réintroduisant une maîtrise socio-politique locale dans ce domaine. On peut suggérer ici deux spécificités de l'agglomération dunkerquoise comme territoire urbano-industriel. D'une part, la ville s'est industrialisée dans la deuxième moitié $\mathrm{du} \mathrm{XX}^{\mathrm{e}}$ siècle en empiétant sur un milieu naturel constitutif de l'identité patrimoniale urbaine, symbolisé par une véritable rupture entre la ville et son front de mer. Ce milieu naturel s'est en quelque sorte immiscé à nouveau dans le fonctionnement de la ville avec la crise industrielle et le réaménagement indispensable des friches qu'elle a provoqué. En ce sens, le premier enseignement à tirer de ce cas est bien ici la nécessité pour les villes-usines de reconsidérer l'importance du milieu naturel initial de la ville, et plus généralement des interactions désormais incontournables entre ce milieu naturel et le milieu socio-économique. D'autre part, cette réintroduction du milieu naturel doit également beaucoup aux revendications des populations locales en faveur d'un environnement sain. En effet, face aux pollutions industrielles, les habitants sont souvent entrés en opposition, voire en conflit ouvert, avec les positions des industriels et les postures parfois un peu attentistes de certains élus locaux sur la question de la qualité de l'air $^{23}$. Il faut souligner ici l'influence probable dans cette contestation d'une absence initiale de paternalisme dans les relations entre industrie-population-pouvoir politique local, dont on sait qu'il a joué un rôle essentiel dans le fonctionnement et la structuration des villes-usines. Cette reconnexion de la ville à la nature peut être appréhendée comme le développement d'un nouveau cadre urbain autour d'importantes aménités environnementales, et de la 
valorisation des friches industrielles, notamment celles des centres-urbains longtemps occupées par l'industrie dominante dans les villes-usines. Puisque la rupture entre ville et nature s'est accentuée avec la multiplication dans les anciennes villes-usines de gigantesques friches industrielles (Husser \& Raison, 2015), la constitution d'une nouvelle interface ville-nature apparaît prépondérante. Elle suppose la prise en charge des modifications paysagères issues de l'activité industrielle, la création d'espaces verts, la promotion d'une approche écologique des activités humaines, etc. Néanmoins, l'exemple de Dunkerque permet de souligner que cette conciliation ville et nature dans la quête d'un renouveau urbain - ne peut l'être qu'à partir d'une véritable stratégie territoriale portée par une pluralité d'acteurs. Il s'agit d'une recomposition territoriale éminemment politique qui dépend des interactions sociales entre les acteurs territoriaux. Et elle n'est souvent pas loin d'une tentative d'expression d'identités régionales, urbaines, et notamment naturelles qui furent décimées par l'activité usinière. Les espaces de loisirs que ce nouveau rapport ville-nature permet de créer, constituent par ailleurs des opportunités de mixité sociale.

En second lieu, l'engagement du territoire dunkerquois dans une démarche collective d'écologie industrielle apparaît comme une dimension non moins importante de la stratégie d'attractivité territoriale. En effet, les crises successives n'ont pas pour autant décimé l'ensemble du tissu industriel local. Certaines activités industrielles, notamment dans la sidérurgie et la cimenterie sont encore présentes. De fait, le processus de recomposition territoriale des villes-usines ne peut donc occulter l'impératif d'une conciliation de l'activité industrielle aux enjeux de transition écologique. Cela suggère un changement de paradigme économique avec l'interrogation sur la poursuite de la croissance économique à moyen et long terme pour la lutte contre le changement climatique et la réduction de la biodiversité. Si, à l'image de beaucoup de villes-usines, un redéploiement vers le secteur tertiaire a été opéré au sein du tissu économique dunkerquois, il n'en reste pas moins que les acteurs, politiques, industriels et institutionnels, ont construit une stratégie collective de minimisation des déchets résiduels et flux issus de l'industrie, constitutive désormais de l'identité industrielle dunkerquoise. De plus, même si les spécificités industrielles sont incontestablement différentes dans les villes-usines (avec en particulier pour ces dernières une dépendance très forte vis-à-vis des secteurs en déclin ou disparus, comme les mines), les acteurs locaux de ces territoires spécifiques pourraient sans doute tirer des enseignements de cette stratégie. On peut faire l'hypothèse en effet que l'écologie industrielle pourrait aider à refonder les bases d'une articulation entre l'industrie existante et les enjeux précédemment évoqués et désormais incontournables. Soulignons enfin que ces initiatives s'inscrivant dans le cadre de l'écologie industrielle, comme le montre l'exemple dunkerquois, vont dans le sens d'une réduction des pollutions. De plus, elles rejoignent ainsi potentiellement les politiques urbaines d'amélioration de la qualité de vie et de prise en compte des contraintes environnementales, dimension essentielle d'une reconstruction du rapport ville-nature.

En définitive, les enseignements que l'on peut tirer de l'évolution du territoire urbanoindustriel dunkerquois pour les stratégies de re-dynamisation socio-économique des villes-usines sont multiples. Au-delà des deux leviers que nous avons soulignés ici - la reconnexion de la ville à la nature et le développement de l'écologie industrielle - la recomposition des villes-usines peut bien relever de nouvelles conceptions du développement territorial, notamment du point de vue de la gouvernance. En effet, 
cette restructuration urbaine renvoie à une appropriation collective et/ou une compréhension mutuelle des enjeux par l'ensemble des acteurs territoriaux, des modalités de mise en œuvre des actions résolument ancrées dans une forme de complémentarité de ressources et de compétences, notamment entre les acteurs publics et privés. Elle relève aussi de la prise en compte des conflits de voisinage et d'usage entre l'industrie et les populations locales, c'est-à-dire de la construction d'un nouveau rapport et/ou de nouveaux liens de proximité entre les usagers d'un même espace urbain. Les villes-usines, précisément parce qu'elles incarnent par leur trajectoire historique cette volonté humaine de façonner la ville à partir d'une usine en se déconnectant du milieu naturel, pourraient constituer un terrain propice à une prise de conscience de l'attachement intrinsèque (et risqué !) de la ville à son milieu naturel pour la reconstruction d'une nouvelle attractivité.

\section{BIBLIOGRAPHIE}

Aubert D., 2001, « Photographie et utopie industrielle : Pittsburgh au début du XXe siècle », Revue françaises d'études américaines, Vol.3, n89, p.33-422.

Bacqué M.-H., Bellanger E., Rey H., 2018, Banlieues populaires Territoires, sociétés, politiques. La Tour d'Aigues, Editions de l'Aube, Bibliothèque des territoires, 382p.

Béal V., Fol S., Rousseau M., 2016, « De quoi le 'smart shrinkage' est-il le nom ? Les ambiguïtés des politiques de décroissance planifiée dans les villes américaines ", Géographie, économie, société, Vol. $18, \mathrm{n}^{\circ} 2, \mathrm{p} .211-234$.

Beaurain C., Frère S., Flanquart H., 2017, « Un territoire industriel confronté aux enjeux environnementaux », in Frère S., Flanquart H. (dir), La ville et ses Risques : habiter Dunkerque, Environnement et Sociétés, Presses Universitaires du Septentrion, p.43-70.

Beaurain, C., Varlet, D. 2015, « Régulation des interactions au sein d'un réseau territorialisé d'entreprises dans le cadre de l'écologie industrielle. » Revue d'économie industrielle, n¹52, p.173206.

Beaurain C., 2014, « La nature en ville contre l'industrie, tout contre... L'exemple de l'agglomération dunkerquoise. ", in Bourdeau-Lepage L., Vidal R., Nature en Ville, Attentes citadines et Actions Publiques, Paris, Ed. Editopics, Collections Séries, p.76-96.

Beaurain C., 2008, « La construction d'un territoire à partir des ressources environnementales : l'exemple de l'agglomération dunkerquoise ». Géographie, Economie, Société, Vol.10, n³, p.365-384.

Beaurain C. (dir.), Flanquart H., Hellequin A.P., Calvo-Mendieta I, Frère S., Le Blanc Antoine, Gonthier F., Bernard J., 2009, « De la perception individuelle des risques industriels à l'action collective en faveur de la sécurité industrielle : une approche territoriale », programme de recherche financé par la Fondation pour une Culture de la Sécurité Industrielle (FONCSI), novembre, $182 \mathrm{p}$. 
Beaurain C., Maillefert M., Liefooghe C., Zuindeau B., 2006, «Politiques territoriales de l'environnement et développement économique : une approche territoriale », Rapport de recherche, Ministère de l'Ecologie et du Développement Durable, juin, 187 p.

Bergeron L., Dorel-Ferré G., 1996, Le patrimoine industriel, un nouveau territoire, Paris, LIRIS, 127p.

Bourdeau-Lepage L., 2017, Ville et nature, vers une nouvelle alliance? , in P. Hamman, « Ruralité, Nature, et environnement », Paris, ERES, p.359-374.

Castells M., Godard F., 1974, Monopolville. L'entreprise, l'Etat, l'urbain, Paris, Mouton, 496p.

Chertow M., 2007, « Uncovering Industrial Symbiosis », Journal of Industrial Ecology, vol. 11, n²1, p.11-30.

Del Biondo L., Edelblutte S., 2016, « Le paysage des anciennes villes-usines européennes : un nouveau patrimoine entre négation, alibi, reconnaissance et complexité des jeux d'acteurs. » Annales de Géographie, ${ }^{\circ} 711$, p.466-489.

Del Biondo L., 2014, Les stratégies de recomposition urbaine soutenable des anciens territoires industrialo-urbains, Thèse de doctorat, Université de Lorraine, $425 \mathrm{p}$.

Doyen J-P., 1983, « Les villes-usines de la moyenne Moselle », Annales de la Société d'Émulation des Vosges, p.52-71.

Edelblutte S., 2014, « Reconversion industrielle ou redéveloppement territorial ? L'exemple de Thaon-les-Vosges, ancienne ville-usine textile lorraine ", Géoconfluences, 2014, mis en ligne le 2 décembre 2014, consulté le 29 novembre 2017, URL https://lc.cx/Yz24

Edelblutte S., 2010b, Paysages et territoires de l'industrie en Europe : genèse, crise et renouveau, Paris, Ellipses, 272p.

Edelblutte S., 2010a, « La reconversion des anciennes villes-usines européennes ou la question de la survie urbaine. ", Bulletin de l'Association des géographes français, Vol.87, n³, p.353-367.

Edelblutte S., 2009, «Que sont devenues les villes-usines ? Réflexion à partir du cas lorrain. » in C. Vallat - dir. Dufaux F., Lehman-Frisch S. (coord), Pérennité urbaine, ou la ville par-delà des métamorphoses, Vol.3 : Essences, Paris, L'Harmattan, p.137-153.

Fol S., Cunninggham-Sabot E., 2010, « Déclin urbain et Shrinking Cities : une évaluation critique des approches de la décroissance urbaines. », Annales de Géographie Vol. 4, p.359-383.

Fortin, M.-J., \& Gagnon, C. (2006). « Interpreting major industrial landscapes: Social follow-up on meanings, the case of two aluminium smelters, Alcan (Alma, Canada) and Pechiney (Dunkirk, France). » Environmental Impact Assessment Review, Vol 26, n8, p.725-745.

Georgeault L., 2015, «L'écologie industrielle et son pilotage dans la construction d'une politique publique d'aménagement du territoire en France », in Mat N., Cerceau J., Alix Y., (Coord). Économie Circulaire et Écosystèmes Portuaires. Collection Les Océanides, p.57-72.

Husser C., Raison S., 2015, « Dunkerque : la reconversion de terrains portuaires », Annales des Mines-Réalités Industrielles Vol.4, p.66-71

Jalabert G., Grégoris M., 1987, « Turin : de la ville-usine à la technopole », Annales de Géographie Vol. 96, n538, p.680-704.

Joan J.M., Méha C., Hellequin P., Beaurain C., 2017, « Genèse d'un territoire industrialoportuaire », in Frère S., Flanquart H. (dir), La ville et ses Risques : habiter Dunkerque, Environnement et Sociétés, Presses Universitaires du Septentrion, p.25-42. 
Krugman P. R., 1991, Geography and Trade, Leuven University Press and The MIT Press, Cambridge, $156 \mathrm{p}$.

Krugman P. R., 1993, « On the number and location of cities », European Economic Review, Vol. 37, $\mathrm{n}^{\circ}(2-3), \mathrm{p} .293-298$.

Latour B., 1999, Politiques de la nature. Comment faire entrer les sciences en démocratie. La Découverte, Paris, $382 \mathrm{p}$.

Le Brun J., Lasseray M., 1936, « Un paysage industriel, Le Creusot. », L'information Géographique, 1(4), 184-186 : https://doi.org/10.3406/ingeo.1936.6557

Luxembourg C., 2008, Villes en transition : les avatars de l'industrie dans les villes françaises petites et moyennes (Blagnac, Bourges, Gennevilliers, Le Creusot, Valenciennes). Thèse de Géographie, Université de Nanterre-Paris X, $337 \mathrm{p}$.

Luxembourg C, 2014, Métamorphose des villes industrielles. Vivre dans la ville désindustrialisée. Paris, L'Harmattan, 178p.

Porteous J., 1970, « The Nature of Company Town. » Transactions of The Institute of British Geographers, Vol. 51, p.127-142.

Picon M., 2014, Autour de l'Orne industrieuse: Paysages hérités, mémoire de master, Ingénierie de l'environnement, École Supérieure des Géomètres et Topographes, 223 p.

Raggi P, 2019, La désindustrialisation de la Lorraine du fer. Pais, Classiques Garnier, 506p.

Roth, L. 2018, Espaces fragiles. Construction scientifique, dynamiques territoriales et action publique. Presses Universitaires Blaise Pascal, Clermont-Ferrand, 260p.

Ryan, B. D., and D. Campo., 2013, « Autopia's End: The Decline and Fall of Detroit's Automotive Manufacturing Landscape. » Journal of Planning History Vol 12, $n^{\circ} 2$, p.95-132.

Teulon F., 2010, Croissance, Crises et développement, Paris, PUF, 392 p.

Varlet D., 2012, Enjeux, potentialités et contraintes de l'écologie industrielle : le cas de Dunkerque, Thèse de doctorat, Sciences de Gestion, Université du Littoral Côte d'Opale, 307p.

Verley P., 2016, La première révolution industrielle, $2^{\mathrm{e}}$ Edition, Armand Colin, $132 \mathrm{p}$.

\section{NOTES}

1. D'une certaine manière, cette évolution des villes-usines vient également relativiser le caractère opératoire du principe de "destruction créatrice ", cher à J.A. Schumpeter, dans la mesure où la disparition de secteurs entiers de l'économie n'est que très difficilement compensée par l'émergence de nouvelles activités.

2. Le développement de la ville du Creusot est étroitement lié à l'industrie sidérurgique très paternaliste de la dynastie Schneider

3. Ce cosmopolitisme s'explique bien sûr également par le positionnement spécifique de cette ville sur la frontière tracée en 1871.

4. Dans ce domaine, rappelons que les Etablissements Publics Fonciers ont joué un rôle essentiel, à travers leur politique d'acquisition foncière, de conduite des opérations d'aménagement ou encore de conseil auprès des collectivités locales.

5. La ville de Dunkerque compte aujourd'hui un peu plus de 70.000 habitants. Avec 17 autres communes du territoire, elle constitue la Communauté Urbaine de Dunkerque, créée en 1969, et atteignant une population de près de 210.000 habitants. 
6. Auquel il faut ajouter la présence d'un site nucléaire de grande envergure avec la centrale de Gravelines, la plus importante en Europe de l'Ouest.

7. Les plages et les digues de mer (station balnéaire de Malo-les-bains et digue du Braek), qui s'étalent sur près de $12 \mathrm{~km}$, ont largement été appropriées par les habitants pour des usages récréatifs (sports de voile, sports de plage, pêche...) et la recherche d'un contact quotidien à la nature, en même temps qu'elles assurent un tourisme balnéaire important durant les mois d'été. La présence massive des espaces dunaires protégés contribue également à la qualité et au caractère sauvage du paysage naturel. À l'ouest de l'agglomération, plusieurs espaces naturels font ainsi l'objet d'un classement: zone naturelle, Zone Naturelle d'Intérêt Faunistique et Floristique (ZNIEFF 1 et 2), Grands sites de France (site des deux caps : Gris nez et Blanc nez sur le littoral de la Côte d'Opale), auxquels s'ajoute un parc naturel régional (le PNR des côtes et marais d'Opale) ou encore des zones humides du Platier d'Oye. La trame verte / trame bleue à l'échelle de l'agglomération (près de 5000ha), outre les espaces de nature «classiques » (bases de loisirs, parcs d'agglomération, parcs urbains, chemins verts, cours d'eau, zones humides et cœurs de nature), intègre également des espaces classés en Natura 2000 "mixte: terre et mer » et en Natura 2000 « mer».

8. Les principaux polluants émis sur la zone sont le dioxyde de soufre (SO2), les hydrocarbures aromatiques polycycliques (HAP), l'oxyde d'azote (NOx), les composés organiques volatils non méthaniques (COVMN), le plomb et les poussières. Les sites sidérurgiques, de l'industrie chimique et de l'industrie pétrolière sont les principaux émetteurs de ces polluants. L'agglomération recense également une quinzaine de sites classés Seveso pour les risques industriels, dont la plupart en Seveso « seuil haut».

9. La construction d'un réseau autoroutier le long du littoral à la fin du XXe a encore accentué ce caractère stratégique de l'activité portuaire.

10. Rappelons que le capitalisme monopoliste d'État a été défini dans les années 1970 comme ce stade où les alliances entre firmes visant à la création de monopoles dans certains secteurs ont été relayées par une intervention importante de l'État dans de multiples domaines, dans le cadre d'une internationalisation des logiques productives. Ces domaines touchent soit au capital financier soit au capital productif dans sa dimension interne (rationalisation des process de production) soit dans sa dimension externe (mobilité de la main d'œuvre, aménagement de l'espace urbain) pour aboutir en définitive à une logique sociale qui repose à la fois sur l'accumulation des capitaux et sur la concentration des moyens de production et de la force de travail. Dans cette logique, l'appareil d'État et la grande entreprise participent l'un et l'autre à la régulation des structures socio-économiques et à la résolution des contradictions pouvant s'exprimer entre les multiples intérêts.

11. Entre 1962 et 1975, 12500 emplois ont été créés dans le secteur de la sidérurgie et les effectifs d'Usinor sont passés de 500 à 11.000 salariés.

12. Le port autonome de Dunkerque est aujourd'hui le $3^{\text {ème }}$ port industriel de France, avec un trafic qui a atteint en 2018 les 51,6 millions de tonnes (20\% marchandises diverses, $25 \%$ vracs solides, $10 \%$ vracs liquides, $20 \%$ trafic transManche), auxquels s'ajoute le trafic conteneurs : $422000 \mathrm{EVP})$.

13. 15 millions de $\mathrm{m} 3$ de sables ont ainsi été dégagés pour permettre la construction de l'usine et d'infrastructures routières et ferroviaires, et le sol naturel a dû être relevé de 9 mètres : Au total, ce sont ainsi 800.000 tonnes de béton qui ont ainsi été utilisées pour l'ensemble de cette construction.

14. Entre 1962 et 1975, la population de la Communauté Urbaine s'est accrue de 4,7\% par an, et cette croissance est directement liée à l'industrialisation car elle a surtout été concentrée dans les villes accueillant la population ouvrière de la sidérurgie : Grande-Synthe, Dunkerque, FortMardyck, Loon-plage. La ville de Grande-Synthe est ainsi passée durant cette période de 3000 habitants à plus de 20 000. A partir des années 1980, les communes proches des industries ont 
connu une stagnation voire un déclin démographique, tandis que la croissance de la population se reportait sur les petites communes rurales plus éloignées de l'industrie (Joan, Meha, Hellequin, Beaurain, 2017).

15. Notamment la Zone à urbaniser en priorité des Nouvelles Synthes à Grande-Synthe, au début des années 1960, et la Zone d'aménagement concerté du Courghain à Dunkerque.

16. Arcelor-Mittal compte aujourd'hui un peu plus de 4.000 salariés, contre 11.000 au milieu des années 1970.

17. Sur la difficulté à saisir les attentes et désirs des populations citadines à l'égard de l'introduction de la nature en ville, cf. L.Bourdeau-Lepage (2017).

18. Citons par exemple la «Charte pour la qualité de l'environnement» (1990), le "Schéma d'environnement industriel » (1993), le «Livre blanc de l'environnement » (1994), les « Contrats d'agglomération» de 1991, 1994 et 2000. A partir de 2007, le contrat d'agglomération a été intégré dans l'Agenda 21 local.

19. Au titre de ces dispositifs on peut citer par exemple la création dans les années 1990 d'un réseau local de surveillance et de mesure de la qualité de l'air, d'une structure de concertation (le Secrétariat pour la Prévention des Pollutions Industrielles), d'un plan de prévention de l'atmosphère et, plus récemment, d'un plan de prévention des risques industriels.

20. Ce terme indique qu'il s'agit d'une chaleur (énergie) résiduelle issue d'un procédé industriel et non récupérée, car le plus souvent rejetée dans l'atmosphère.

21. A ce jour, ce réseau permet de chauffer près de 16000 équivalents logements, soit 50.000 équivalents habitants. En 2020, il est prévu d'augmenter sa capacité de 12.000 équivalents logements supplémentaires. Il permet d'éviter l'émission de 30.000 tonnes/an CO2 et la consommation de 11000 tep. Il a créé 8 emplois directs et autant d'emplois induits.

22. Prix européen des villes durables en 1996, agenda 21 local signé en 2001 dans le cadre du contrat d'agglomération

23. Le rôle central des délibérations collectives à propos de la qualité de l'air (et du risque industriel) dans la réintroduction du rapport à la nature n'est pas sans rappeler la distinction opérée par B. Latour (1999) entre les « objets sans risque » (ou « objets chauves »), qui renvoient à une distinction nette entre nature et politique, et les «attachements risqués » que de multiples fils (ou cheveux) rattachent au politique, par le biais de la production technique et des usines par exemple. Ces attachements font l'objet de multiples discussions et controverses, précisément en vertu de cette connexion intrinsèque avec le monde des humains. D'où l'impossibilité qu'il y a selon cet auteur de distinguer nettement nature et politique (social) si l'on prend en compte ces " attachements ", comme le traduit l'intérêt porté à leurs conséquences sur la société. Dans la logique de cet argumentaire, le terme de "nature » que nous utilisons pour notre propos ne serait toutefois pas réellement pertinent.

\section{RÉSUMÉS}

Fortement dépendantes de secteurs industriels en déclin ou disparus, les villes-usines sont confrontées depuis quelques années à des problématiques de reconversion et de (ré)attractivité. Dans ce cadre, la dimension environnementale, largement sacrifiée pendant les années de croissance, constitue un enjeu majeur, renforcé encore par la perspective de la transition écologique et sociale. Dans cet article, nous tentons de formuler quelques pistes de réflexion pour esquisser les contours d'une articulation de ces villes-usines à une reconsidération du rôle et de 
l'importance de l'environnement naturel dans le développement territorial. Pour cela, nous prenons appui sur l'exemple du territoire urbano-industriel de l'agglomération dunkerquoise, dont nous tirons des enseignements mis en perspective avec le cas particulier des villes-usines.

Highly dependent on declining or disappeared industrial sectors, company towns have been faced in recent years with problems of renovation and (re)attractiveness. In this context, the environmental dimension, which was largely surrendered during the years of growth, is a major challenge, further reinforced by the perspective of ecological and social transition. In this article, our main objective is to expose some avenues for reflection to outline the contours of an articulation of these company towns with a reconsideration of the role and importance of the natural environment in their territorial development. In fact, we use the example of the urbanindustrial territory of Dunkerque (France), from which we draw lessons put into perspective with the particular case of factory towns.

Die von rückläufigen oder schwindenden Industriebereichen stark abhängigen Fabrikstädte sind seit einigen Jahren mit Problemen der Umstellung und (Wieder-) Attraktivität konfrontiert. In diesem Zusammenhang stellt die Umweltdimension, die in den Jahren des Wachstums weitgehend geopfert wurde, eine große Herausforderung dar, die durch die Aussicht auf einen ökologischen und sozialen Wandel noch verstärkt wird. In diesem Artikel versuchen wir, einige Gedankengänge $\mathrm{zu}$ formulieren, um die Konturen einer Artikulation dieser Fabrikstädte zu skizzieren und die Rolle und Bedeutung der natürlichen Umwelt für die territoriale Entwicklung zu überdenken. Dafür stützen wir uns auf das Beispiel des städtischen Industriegebiets der Agglomeration Dünkirchen, aus dem wir Lehren ziehen, die mit dem besonderen Fall der Fabrikenstädte in Beziehung gesetzt werden.

\section{INDEX}

Mots-clés : Villes-Usines - Aménagement - Nature - Écologie Industrielle - Dunkerque

Keywords : Factory town - Planning - Nature - Industrial Ecology - Dunkerque

Schlüsselwörter : Fabrikstädte - Planung - Natur - Industrieökologie - Dünkirchen

\section{AUTEURS}

\section{CHRISTOPHE BEAURAIN}

Professeur d'économie-aménagement, Université de Limoges, CNRS, GEOLAB, UMR 6042, Université de Limoges, F-87036 Limoges, France christophe.beaurain@unilim.fr

\section{CHEDRAK DE ROCHER CHEMBESSI}

Doctorant, Université de Limoges, CNRS, GEOLAB UMR 6042 - Université Laval, Centre de Recherche en Aménagement du Territoire et Développement

Régionalchedrak.chembessi@unilim.fr / chedrak-sylvain-de-rocher.chembessi.1@ulaval.ca 